CUBO A Mathematical Journal Vol.15, № 02, (21-31). June 2013

\title{
Nonnegative solutions of quasilinear elliptic problems with sublinear indefinite nonlinearity 1
}

\author{
WeIHUI WANG ${ }^{a}$ AND ZUODONG YANG ${ }^{a, b}$ \\ a Institute of Mathematics, School of Mathematical Sciences, \\ Nanjing Normal University, \\ Jiangsu Nanjing 210046, China. \\ b College of Zhongbei, \\ Nanjing Normal University, \\ Jiangsu Nanjing 210046, China. \\ 335348332@qq.com zdyang_jin@263.net
}

\begin{abstract}
We study the existence, nonexistence and multiplicity of nonnegative solutions for the quasilinear elliptic problem

$$
\begin{cases}-\triangle_{p} u=a(x) u^{q}+\lambda b(x) u^{r}, & \text { in } \Omega \\ u=0, & \text { on } \partial \Omega\end{cases}
$$

where $\Omega$ is a bounded domain in $\mathbf{R}^{\mathrm{N}}, \lambda>0$ is a parameter, $\triangle_{\mathfrak{p}}=\operatorname{div}\left(|\nabla \mathbf{u}|^{\mathrm{p}-2} \nabla \mathbf{u}\right)$ is the $p$-Laplace operator of $u, 1<p<N, 0<q<p-1<r \leq p^{*}-1, a(x), b(x)$ are bounded functions, the coefficient $b(x)$ is assumed to be nonnegative and $a(x)$ is allowed to change sign. The results of the semilinear equations are extended to the quasilinear problem.
\end{abstract}

\footnotetext{
${ }^{1}$ Project Supported by the National Natural Science Foundation of China(Grant No.11171092). Project Supported by the Natural Science Foundation of the Jiangsu Higher Education Institutions of China (Grant No.08KJB110005)
} 


\section{RESUMEN}

Estudiamos la existencia, no existencia y multiplicidad de soluciones no negativas del problema elíptico cuasi-lineal

$$
\begin{cases}-\triangle_{p} u=a(x) u^{q}+\lambda b(x) u^{r}, & \text { in } \Omega \\ u=0, & \text { on } \partial \Omega\end{cases}
$$

donde $\Omega$ es un dominio acotado en $\mathbf{R}^{\mathrm{N}}, \lambda>0$ es un parámetro, $\triangle_{\mathrm{p}}=\operatorname{div}\left(|\nabla \mathbf{u}|^{\mathrm{p}-2} \nabla \mathbf{u}\right)$ es el operador $p$-Laplaciano de $u, 1<p<N, 0<q<p-1<r \leq p^{*}-1, a(x)$, $b(x)$ son funciones acotadas, el coeficiente $b(x)$ se supone que es no negativo y $a(x)$ se le permite cambiar de signo. Los resultados de las ecuaciones semilineales se extienden a el problema cuasi-lineal.

Keywords and Phrases: Nonnegative solutions; quasilinear elliptic problems; sublinear indefinite nonlinearity; Existence and nonexistence.

2010 AMS Mathematics Subject Classification: 35J50, 35J55, 35J60. 


\section{Introduction}

Let us consider the problem

$$
\begin{cases}-\triangle_{p} u=a(x) u^{q}+\lambda b(x) u^{r}, & \text { in } \Omega \\ u=0, & \text { on } \partial \Omega\end{cases}
$$

where $\Omega \subset \mathbf{R}^{N}$ is a smooth bounded domain, $\lambda>0,1<p<N, 0<q<p-1<r \leq p^{*}-1$, $\mathrm{p}^{*}=\frac{\mathrm{Np}}{\mathrm{N}-\mathrm{p}}, \mathrm{b}(\mathrm{x}) \geq 0, \mathrm{a}(\mathrm{x})$ change its sign, $\triangle_{\mathrm{p}}=\operatorname{div}\left(|\nabla \mathrm{u}|^{\mathrm{p}-2} \nabla \mathrm{u}\right)$ is the $\mathrm{p}$-Laplace operator of $u$. Equations of the above form are mathematical models occuring in studies of the p-Laplace equation, generalized reaction-diffusion theory $([7])$, non-Newtonian fluid theory, and the turbulent flow of a gas in porous medium([8]). In the non-Newtonian fluid theory, the quantity $p$ is characteristic of the medium. Media with $p>2$ are called dilatant fluids and those with $p<2$ are called pseudoplastics. If $p=2$, they are Newtonian fluids.

Recently, A.V.Lair and A.Mohammed in [11] considered the existence and nonexistence of positive entire large solutions of the semilinear elliptic equation

$$
\triangle u=p(x) u^{\alpha}+q(x) u^{\beta}, \quad 0<\alpha \leq \beta .
$$

Francisco in [1] considered a sublinear indefinite nonlinearity problem of the form

$$
\begin{cases}-\Delta u=a(x) u^{q}+\lambda b(x) u^{p}, & \text { in } \Omega \\ u=0, & \text { on } \partial \Omega\end{cases}
$$

where $\Omega$ is a smooth bounded domain in $\mathbf{R}^{\mathrm{N}}, \lambda \in \mathrm{R}, 0<\mathrm{q}<1<\mathrm{p}<\mathrm{r} \leq 2^{*}-1, \mathrm{~b}(\mathrm{x}) \geq 0$, $a(x)$ change its sign. For more results we refer the reader to the works [12-15] and the references therein.

In recent years, the existence and uniqueness of the positive solutions for the single quasilinear elliptic equation with eigenvalue problems

$$
\left\{\begin{array}{l}
\operatorname{div}\left(|\nabla u|^{p-2} \nabla u\right)+\lambda f(u)=0 \quad \text { in } \Omega \\
u(x)=0 \quad \partial \Omega
\end{array}\right.
$$

with $\lambda>0, p>1, \Omega \subset \mathbf{R}^{\mathbf{N}}, \mathbf{N} \geq 2$ have been studied by many authors, see [16-23] and the references therein. When $f$ is strictly increasing on $\mathbf{R}^{+}, f(0)=0, \lim _{s \rightarrow 0^{+}} f(s) / s^{p-1}=0$ and $f(s) \leq \alpha_{1}+\alpha_{2} s^{\mu}, 0<\mu<p-1, \alpha_{1}, \alpha_{2}>0$, it was shown in [16] that there exist at least two positive solutions for Eqs (1.1) when $\lambda$ is sufficiently large. If $\lim _{s \rightarrow 0^{+}} \inf f(s) / s^{p-1}>0, f(0)=0$ and the monotonicity hypothesis $\left(f(s) / s^{p-1}\right)^{\prime}<0$ holds for all $s>0$. It was also shown in [17] that problem (1.1) has a unique positive large solution and at least one positive small solution when $\lambda$ is large if $f$ is nondecreasing; there exist $\alpha_{1}, \alpha_{2}>0$ such that $f(s) \leq \alpha_{1}+\alpha_{2} s^{\beta}, 0<\beta<$ $p-1 ; \lim _{s \rightarrow 0^{+}} \frac{f(s)}{s^{p-1}}=0$, and there exist $T, Y>0$ with $Y \geq T$ such that

$$
\left(f(s) / s^{p-1}\right)^{\prime}>0 \text { for } s \in(0, T)
$$


and

$$
\left(\mathrm{f}(\mathrm{s}) / \mathrm{s}^{\mathrm{p}-1}\right)^{\prime}<0 \text { for } \mathrm{s}>\mathrm{Y} .
$$

Yang and $\mathrm{Xu}$ in [10] established the existence for quasilinear elliptic equation

$$
\left\{\begin{array}{l}
-\triangle_{p} u=a(x)\left(u^{m}+\lambda u^{n}\right), \quad x \in \mathbf{R}^{N} \\
u>0, \quad x \in \mathbf{R}^{N} \\
u \rightarrow 0, \quad|x| \rightarrow \infty
\end{array}\right.
$$

where $0<\mathrm{m}<\mathrm{p}-1<\mathrm{n}$, they proved there exists a $\lambda^{*}>0$ such that (1.2) has a positive solution for $0<\lambda<\lambda^{*}$.

The quasilinear elliptic equations when $a(x) \equiv b(x) \equiv 1$ was considered in [2], although here under some restrictions on the $p, q$ in the critical case $r=p^{*}-1$. Problems of local "superlinearrity" and "sublinearity" for the $p$ - Laplace problem was considered in [3]. A class of quasilinear elliptic equations are study in [4]. For more results we refer the reader to the works [5-6] and the references therein.

Motivated by the results of the above papers. In this paper, we consider the quasilinear elliptic equations $\left(\mathrm{P}_{\lambda}\right)$. We modify the method developed Francisco Odair de Paiva in [1] and extend the results a quasilinear elliptic equation $\left(\mathrm{P}_{\lambda}\right)$, and complement results in $[2-4,10]$.

The paper is organized as follows. In section 2 , we recall some facts that will be needed in the paper, and give the main results. In section 3 , we give the proofs of the main results in this paper.

\section{Main results and Preliminary}

Let us first consider the following parameterized elliptic problems

$$
\begin{cases}-\triangle_{p} u=a(x) u^{q}+\lambda b(x) u^{r}, & \text { in } \Omega \\ u \geq 0, & \text { in } \Omega \\ u=0, & \text { on } \partial \Omega\end{cases}
$$

where $\Omega$ is a bounded domain in $\mathbf{R}^{\mathrm{N}}, \lambda>0$ is a parameter, $1<\mathrm{p}<\mathrm{N}, 0<\mathrm{q}<\mathrm{p}-1<\mathrm{r} \leq$ $p^{*}-1, a(x), b(x)$ are bounded functions, the coefficient $b(x)$ is assumed to be nonnegative and $a(x)$ is allowed to change sign. Because that $a(x)$ changes sign in $\Omega$, so the Maximum principal is not applicable. Then, define

$$
F_{\lambda}(u)=\frac{1}{p} \int_{\Omega}|\nabla u|^{p}-\frac{1}{q+1} \int_{\Omega} a(x)\left(u^{+}\right)^{q+1}-\frac{\lambda}{r+1} \int_{\Omega} b(x)\left(u^{+}\right)^{r+1}, u \in W_{0}^{1, p}(\Omega)
$$

We know that $F_{\lambda}(u)$ is well define in $W_{0}^{1, p}(\Omega)$ and is of $C_{0}^{1}(\bar{\Omega})$ 
Definition 2.1. We call $u \in W_{0}^{1, p}(\Omega)$ is a weak solution of $\left(Q_{\lambda}\right)$, if $u$ is a critical points of $F_{\lambda}(u)$.

Throughout this paper, we always suppose that

$\left(H_{1}\right)$ There exist $\lambda>0$, a smooth subdomain $B_{1} \in \Omega_{a}^{+}, m(x) \in L^{\infty}\left(B_{1}\right)$ with $m(x) \geq 0, m(x) \not \equiv$ $0, \mu>\lambda_{1}\left(B_{1}, m(x)\right)$ such that

$$
a(x) s^{q}+\lambda b(x) s^{r} \geq \mu m(x) s^{p-1}
$$

for a.e. $x \in B_{1}$ and all $s \geq 0$; here $\lambda_{1}\left(B_{1}, m(x)\right)$ denotes the principal eigenvalue of $-\triangle_{p}$ on $W_{0}^{1, p}\left(B_{1}\right)$ for the weight $m(x)$.

$\left(H_{2}\right)$ For any $\lambda>0$, there exists a smooth subdomain $B_{2} \subset \Omega_{a}^{+}, s_{1}>0$ and $\theta_{1}>\lambda_{1}\left(B_{2}\right)$, such that

$$
a(x) s^{q}+\lambda b(x) s^{r} \geq \theta_{1} s^{p-1}
$$

for a.e. $x \in B_{2}$, and all $s \in\left[0, s_{1}\right]$; here $\lambda_{1}\left(B_{2}\right)$ denotes the principal eigenvalue of $-\triangle_{p}$ on $W_{0}^{1, p}(\Omega)$

$\left(F_{1}\right) a(x), b(x) \in L^{\infty}(\Omega)$, and

$$
\begin{aligned}
& \Omega_{a}=\{x \in \Omega: a(x) \geq 0\}, \Omega_{a}^{+}=\{x \in \Omega: a(x)>0\} \\
& \Omega_{a}^{-}=\{x \in \Omega: a(x)<0\}, \Omega_{b}^{+}=\{x \in \Omega: b(x)>0\}
\end{aligned}
$$

are nonempty;

$\left(F_{2}\right) \Omega_{a}^{+}$is open, $\left|\Omega_{\mathrm{a}}^{-}\right|>0$ and $\overline{\Omega_{\mathrm{a}}^{+}} \cap \overline{\Omega_{\mathrm{a}}^{-}}=\emptyset$;

$\left(\mathrm{F}_{3}\right) \operatorname{int}\left(\Omega_{\mathrm{b}}^{+}\right) \neq \emptyset$ and $\mathrm{b} \geq 0$;

$\left(\mathrm{F}_{4}\right) \Omega_{\mathrm{a}}^{+} \subset \Omega_{\mathrm{b}}^{+}$and $\overline{\Omega_{\mathrm{a}}^{+}} \subset \Omega$;

$\left(F_{5}\right) \operatorname{int}\left(\Omega_{a}\right)=\bigcup_{1}^{k} U_{i}, U_{i}$ connected, and $U_{i} \cap \Omega_{a}^{+} \neq \emptyset$.

As a consequence of assumption $\left(F_{5}\right)$, by the Maximum principle, if $u$ is a solution of $\left(Q_{\lambda}\right)$ such that $u$ is nontrivial in the components of $\Omega_{a}$, then $u>0 \operatorname{in} \operatorname{int}\left(\Omega_{a}\right) \supset \Omega_{a}^{+}$.

Definition 2.2. If $u$ is a weak solution of $\left(Q_{\lambda}\right)$ and $u(x)>0$, a.e. $x \in \Omega_{a}^{+}$, then $u \in W_{0}^{1, p}(\Omega)$ is a solution of (1.1).

Let

$$
\lambda^{*}=\sup \{\lambda>0 ;(1.1) \text { has a solution }\}
$$

By a modification of the method given in [1], we obtain the following main results.

Theorem 2.1. Let $0<q<p-1<r \leq p^{*}-1$. Assume that $\left(F_{1}\right)-\left(F_{5}\right)$ hold, then there exists $\lambda^{*} \in(0, \infty)$ such that

(1) for all $\lambda \in\left(0, \lambda^{*}\right)$, problem $\left(P_{\lambda}\right)$ has at least one weak solutions;

(2) for $\lambda=\lambda^{*}$, problem $\left(P_{\lambda}\right)$ has at least one solution; 
(3) for all $\lambda>\lambda^{*}$, problem $\left(\mathrm{P}_{\lambda}\right)$ has no solution.

Theorem 2.2. Let $0<q<p-1<r<p^{*}-1$. Assume that $\left(F_{1}\right)-\left(F_{5}\right)$ hold, then problem $\left(P_{\lambda}\right)$ has at least two solutions for $0<\lambda<\lambda^{*}$.

\section{The proof of main results}

Lemma 3.1. There is $\lambda_{0}>0$ such that for $0<\lambda \leq \lambda_{0}$, problem $\left(P_{\lambda}\right)$ has a solution.

Proof. Let $e$ be the unique positive solution of

$$
\begin{cases}-\triangle_{p} e=1, & \text { in } \Omega \\ e=0, & \text { on } \partial \Omega\end{cases}
$$

Since $0<q<p-1<r$, we can find $\lambda_{0}>0$ such that for all $0<\lambda \leq \lambda_{0}$ there exists $M=M(\lambda)>0$ satisfying

$$
M^{p-1} \geq M^{\mathrm{q}}\|\mathrm{a}\|_{\infty}\|e\|_{\infty}^{\mathrm{q}}+\lambda M^{\mathrm{r}}\|\mathrm{b}\|_{\infty}\|e\|_{\infty}^{\mathrm{r}}
$$

As a consequence, the function Me satisfies

$$
-\triangle_{p}(M e)=M^{p-1} \geq M^{q}\|a\|_{\infty}\|e\|_{\infty}^{q}+\lambda M^{r}\|b\|_{\infty}\|e\|_{\infty}^{r}
$$

Hence $M e$ is a supersolution of $\left(P_{\lambda}\right)$. Then let $\bar{u}=M e$, we have that $\bar{u}$ is a supersolution for $\left(Q_{\lambda}\right)$. Moreover 0 is a solution of $\left(Q_{\lambda}\right)$, so let $\underline{u}=0$ is a subsolution for $\left(Q_{\lambda}\right)$. It follows form the sub-supersolution argument as in [5] or [6] that $\left(\mathrm{Q}_{\lambda}\right)$ has a nonnegative solution in $A=\left\{u \in W_{0}^{1, p}: 0 \leq u(x) \leq\right.$ Me a.e. $\left.x \in \Omega\right\}$. Then let $c=\inf _{A} F_{\lambda}$,

$$
F_{\lambda}(u)=\frac{1}{p} \int_{\Omega}|\nabla u|^{p}-\frac{1}{q+1} \int_{\Omega} a(x)\left(u^{+}\right)^{q+1}-\frac{\lambda}{r+1} \int_{\Omega} b(x)\left(u^{+}\right)^{r+1}, u \in w_{0}^{1, p}(\Omega),
$$

there exist $u_{\lambda} \in A$ such that $c=\inf _{A} F_{\lambda}\left(u_{\lambda}\right)$ and $u_{\lambda}$ is a solution of $\left(Q_{\lambda}\right)$. Also $u_{\lambda}$ solves $\left(P_{\lambda}\right)$ if $u_{\lambda}>0$ a.e. $x \in \Omega_{a}^{+}$.

By contradiction, suppose that $u_{\lambda} \equiv 0$ a.e. $x \in \Omega_{a}^{+}$, let $\varphi \in C_{c}^{\infty}\left(\Omega_{a}^{+}\right)$be nonnegative and nontrivial, then for sufficiently small $s>0, u_{\lambda}+s \varphi \in A$

$$
\begin{gathered}
F_{\lambda}\left(u_{\lambda}+s \varphi\right)=F_{\lambda}\left(u_{\lambda}\right)+F_{\lambda}(s \varphi) \\
=F_{\lambda}\left(u_{\lambda}\right)+\frac{s^{p}}{p}\|\varphi\|^{p}-\frac{s^{q+1}}{q+1} \int_{\Omega} a(x) \varphi^{q+1}-\frac{\lambda s^{r+1}}{r+1} \int_{\Omega} b(x) \varphi^{r+1}
\end{gathered}
$$

Then we have $F_{\lambda}\left(u_{\lambda}+s \varphi\right)<F_{\lambda}\left(u_{\lambda}\right)$, if $s>0$ is small enough, however this contradicts that the infimum $c=\inf F_{\lambda}$ is achieve at $u_{\lambda}$. So $u_{\lambda}>0$ a.e. $x \in \Omega_{a}^{+}$and is a solution of $\left(P_{\lambda}\right)$.

Lemma 3.2. $\left(P_{\lambda}\right)$ has a solution for all $\lambda \in\left(0, \lambda^{*}\right)$. 
Proof. Given $\lambda<\lambda^{*}$, let $\mathfrak{u}_{\bar{\lambda}}$ be a solution of $\left(\mathrm{P}_{\bar{\lambda}}\right)$, with $\lambda<\bar{\lambda}<\lambda^{*}$. Then

$$
-\triangle_{\mathfrak{p}} \mathfrak{u}_{\bar{\lambda}}=\mathrm{a}(\mathrm{x}) \mathrm{u}_{\bar{\lambda}}^{\mathrm{q}}+\bar{\lambda} \mathrm{b}(\mathrm{x}) \mathrm{u}_{\bar{\lambda}}^{\mathrm{r}} \geq \mathrm{a}(\mathrm{x}) \mathrm{u}_{\bar{\lambda}}^{\mathrm{q}}+\lambda \mathrm{b}(\mathrm{x}) \mathrm{u}_{\bar{\lambda}}^{\mathrm{r}},
$$

which $\mathfrak{u}_{\bar{\lambda}}$ is a supersolution for $\left(\mathrm{P}_{\lambda}\right)$.

Consider $A=\left\{u \in W_{0}^{1, p}: 0 \leq u \leq u_{\bar{\lambda}}\right\}$, there exist $u_{\lambda} \in A$ such that $F_{\lambda}\left(u_{\lambda}\right)=\inf _{A} F_{\lambda}$, and $\mathfrak{u}_{\lambda}$ is a solution of $\left(Q_{\lambda}\right)$, as the proof of Lemma 3.1, $\mathfrak{u}_{\lambda}$ is also the solution of $\left(P_{\lambda}\right)$.

Lemma 3.3. Let $\lambda^{*}=\sup \left\{\lambda>0:\left(P_{\lambda}\right)\right.$ has a solution $\}$, then $0<\lambda^{*}<\infty$.

Proof. Under the assume $\left(H_{1}\right)$, suppose that when $\lambda>0,\left(P_{\lambda}\right)$ has a solution $u_{\lambda} \in$ $W_{0}^{1, p}(\Omega) \cap L^{\infty}(\Omega)$. Consider the eigenvalue problem with weight

$$
\begin{cases}-\triangle_{\mathfrak{p}} v=\mu \mathrm{m}(\mathrm{x})|v|^{\mathrm{p}-2}, & \text { in } \mathrm{B}_{1} ; \\ v=0, & \text { on } \partial \mathrm{B}_{1} .\end{cases}
$$

Since by $\left(\mathrm{H}_{1}\right)$, we have

$$
\int_{\mathrm{B}_{1}}\left|\nabla \mathfrak{u}_{\lambda}\right|^{p-2} \nabla \mathfrak{u}_{\lambda} \nabla \varphi=\int_{\mathrm{B}_{1}}\left(\mathrm{a}(\mathrm{x}) \mathfrak{u}_{\lambda}^{\mathrm{q}}+\lambda \mathrm{b}(\mathrm{x}) \mathfrak{u}_{\lambda}^{\mathrm{r}}\right) \varphi \geq \mu \int_{\mathrm{B}_{1}} \mathrm{~m}(\mathrm{x}) \mathfrak{u}_{\lambda}^{\mathrm{p}-1} \varphi
$$

for all $\varphi \in \mathrm{C}_{\mathbf{c}}^{\infty}(\Omega), \varphi \geq 0$. This show that $\mathfrak{u}_{\lambda}$ is an supersolution of $\left(E_{\mu}\right)$. Furthermore, $\epsilon \varphi_{1}$ is a subsolution of $\left(E_{\mu}\right)$, and $\epsilon \varphi_{1} \leq u_{\lambda}$ for $\epsilon$ small enough.

$$
\int_{\mathrm{B}_{1}}\left|\nabla\left(\epsilon \varphi_{1}\right)\right|^{p-2} \nabla\left(\epsilon \varphi_{1}\right) \nabla \varphi=\lambda_{1} \int_{\mathrm{B}_{1}} \mathrm{~m}(x)\left(\epsilon \varphi_{1}\right)^{p-1} \varphi<\mu \int_{\mathrm{B}_{1}} \mathrm{~m}(\mathrm{x})\left(\epsilon \varphi_{1}\right)^{p-1} \varphi
$$

for $\varphi \in \mathrm{C}_{\mathbf{c}}^{\infty}(\Omega), \varphi \geq 0 ; \varphi_{1}$ is a positive eigenfunction associated to $\lambda_{1}\left(\mathrm{~B}_{1}, \mathrm{~m}(\mathrm{x})\right)$. Then $\left(\mathrm{E}_{\lambda}\right)$ has a solution $v$ with $€ \varphi_{1} \leq v \leq u_{\lambda}$, in particular $v \geq 0, v \not \equiv 0$. For above that $\mu$ is a principal eigenvalue of $-\triangle_{p} u$ on $B$ for the weight $m(x)$. This is contradiction with $\mu>\lambda_{1}\left(B_{1}, m(x)\right)$, and consequently $\lambda^{*}<+\infty$, moreover we can also obtain $\lambda^{*}>0$ to the Lemma 4.1, so, $\lambda^{*} \in(0, \infty)$. Hence, when $\lambda>\lambda^{*}$, problem $\left(\mathrm{P}_{\lambda}\right)$ has no solution.

Lemma 3.4. For $\lambda=\lambda^{*}$, problem $\left(P_{\lambda}\right)$ has at least one solution.

Proof. For the definition of $\lambda^{*}$, let $\lambda_{n}$ be a sequence such that $\lambda_{n} \longrightarrow \lambda^{*}$ with $0<\lambda_{n}<\lambda^{*}, \lambda_{n}$ increasing, let $u_{n}$ be a solution of $P_{\lambda_{n}}$ with $F_{\lambda_{n}}\left(u_{n}\right)<0$ and $F_{\lambda_{n}}^{\prime}\left(u_{n}\right)=0$. We obtain

$$
F_{\lambda_{n}}\left(u_{n}\right)+F_{\lambda_{n}}^{\prime}\left(u_{n}\right) \cdot u_{n} \leq C\left\|u_{n}\right\|,
$$

where

$$
\begin{gathered}
F_{\lambda_{n}}\left(u_{n}\right)=\frac{1}{p} \int_{\Omega}\left|\nabla u_{n}\right|^{p}-\frac{1}{q+1} \int_{\Omega} a(x)\left(u_{n}^{+}\right)^{q+1}-\frac{\lambda_{n}}{r+1} \int_{\Omega} b(x)\left(u_{n}^{+}\right)^{r+1}, \\
F_{\lambda_{n}}^{\prime}\left(u_{n}\right) \cdot u_{n}=\int_{\Omega}\left|\nabla u_{n}\right|^{p}-\int_{\Omega} a(x)\left(u_{n}^{+}\right)^{q+1}-\lambda_{n} \int_{\Omega} b(x)\left(u_{n}^{+}\right)^{r+1}
\end{gathered}
$$

so by Theorem 1.2.1 of [9], we have

$$
\left(\frac{1}{p}+1\right)\left\|u_{n}\right\|^{p} \leq C\left\|u_{n}\right\|^{q+1}+c .
$$


It shows that $u_{n}$ is bounded in $W_{0}^{1, p}$, we have, for a subsequence, $u_{n} \longrightarrow u^{*}$ in $C^{1}(\bar{\Omega})$, hence $u^{*}$ solves $\left(Q_{\lambda}\right)$ in $\Omega$. $u^{*}$ is a solution of $\left.\left(P_{\lambda}\right)\right)$ if $u^{*} \not \equiv 0$ in $\Omega_{a}^{+}$. Assume by contradiction $u^{*} \equiv 0$ in $\Omega_{\mathrm{a}}^{+}$. Under the assume $\left(\mathrm{H}_{2}\right)$, we have

$$
\int_{\mathrm{B}_{2}}\left|\nabla \mathfrak{u}_{n}\right|^{p-2} \nabla \mathfrak{u}_{n} \nabla \varphi=\int_{\mathrm{B}_{2}}\left(\mathrm{a}(x) \mathfrak{u}_{\mathrm{n}}^{\mathrm{q}}+\lambda_{\mathrm{n}} \mathrm{b}(\mathrm{x}) \mathfrak{u}^{\mathrm{r}}\right) \varphi \geq \theta_{1} \int_{\mathrm{B}_{2}} \mathfrak{u}_{\mathrm{n}}^{\mathrm{p}-1} \varphi
$$

for $n$ sufficiently large(so that $0 \leq u_{n}(x) \leq s_{1}$ on $B_{2}$, which is possible since $u_{n} \longrightarrow 0$ uniformly). So that $u_{n}$ is a supersolution for the problem

$$
\begin{cases}-\triangle_{\mathrm{p}} v=\theta_{1}|v|^{\mathrm{p}-2} v, & \text { in } \mathrm{B}_{2} \\ v=0, & \text { on } \partial \mathrm{B}_{2} .\end{cases}
$$

Moreover, since $\theta_{1}>\lambda_{1}$, let $\mathfrak{u}_{\varepsilon}=\varepsilon \varphi_{1}$. We have

$$
-\triangle_{\mathfrak{p}}\left(\mathfrak{u}_{\varepsilon}\right)=\lambda_{1} \mathfrak{u}_{\varepsilon}^{\mathfrak{p}-1}<\theta_{1} \mathfrak{u}_{\varepsilon}^{p-1}
$$

and $\varepsilon \varphi_{1} \leq u_{n}$ on $B_{2}$, for $(\varepsilon>0$ sufficiently small). It shows that the existence of a solution $v$ of $\left(E_{\theta_{1}}\right)$ with $\varepsilon \varphi_{1} \leq v \leq u_{n}$. This is a contradiction with $\theta_{1}>\lambda_{1}$ in assume $\left(H_{2}\right)$. So, $u^{*} \not \equiv 0$ in $\Omega_{a}^{+}$ and is a solution of $\left(\mathrm{P}_{\lambda}\right)$.

Proof of Theorem 2.2. From the Lemma 3.2, we have obtained $\boldsymbol{u}_{\lambda}$ is a local minimizer of $F_{\lambda}(u)$ and is a solution of $\left(P_{\lambda}\right)$. In this section, we hope to find the second solution of the form $v=\mathfrak{u}_{\lambda}+\mathfrak{u}$, by the moutnain pass theorem, where $u$ is a nonnegative solution of

$$
\begin{cases}-\triangle_{p}\left(\mathfrak{u}_{\lambda}+\mathfrak{u}\right)=\mathfrak{a}(x)\left(\mathfrak{u}_{\lambda}+\mathfrak{u}^{+}\right)^{\mathfrak{q}}+\lambda \mathfrak{b}(x)\left(\mathfrak{u}_{\lambda}+\mathfrak{u}^{+}\right)^{r}, & \text { in } \Omega ; \\ \mathfrak{u}=0, & \text { on } \partial \Omega .\end{cases}
$$

$\mathfrak{u} \in \mathbf{W}_{0}^{1, p}(\Omega)$, and $\mathfrak{u} \geq 0$. Then, $\mathfrak{u}_{\lambda}+\mathfrak{u}$ is a second solution of $\left(P_{\lambda}\right)$. Define the associated functional

$$
\begin{gathered}
\mathrm{I}_{\lambda}(\mathfrak{u})=\frac{1}{p} \int_{\Omega}\left|\nabla\left(u_{\lambda}+\mathfrak{u}\right)\right|^{p}-\int_{\Omega} H_{\lambda}(x, u) \\
H_{\lambda}(x, u)=G_{\lambda}\left(x, u_{\lambda}+u^{+}\right)-G_{\lambda}\left(x, u_{\lambda}\right)-g_{\lambda}\left(x, u_{\lambda}\right) u^{+} ; \\
G_{\lambda}(x, u)=\int_{\Omega} g_{\lambda}(x, u) d u ; \quad g_{\lambda}(x, u)=a(x) u^{q}+\lambda b(x) u^{r} .
\end{gathered}
$$

Then, it follows that

$$
\begin{gathered}
\mathrm{I}_{\lambda}(\mathrm{u})=\frac{1}{\mathrm{p}} \int_{\Omega}\left|\nabla\left(\mathrm{u}_{\lambda}+\mathrm{u}\right)\right|^{p}-\frac{1}{\mathrm{q}+1} \int_{\Omega} \mathrm{a}(\mathrm{x})\left[\left(\mathrm{u}_{\lambda}+\mathrm{u}^{+}\right)^{\mathrm{q}+1}-\mathrm{u}_{\lambda}^{\mathrm{q}+1}-(\mathrm{q}+1) \mathrm{u}_{\lambda}^{\mathrm{q}} \mathrm{u}^{+}\right] \\
-\frac{\lambda}{\mathrm{r}+1} \int_{\Omega} \mathrm{b}(\mathrm{x})\left[\left(\mathrm{u}_{\lambda}+\mathrm{u}^{+}\right)^{\mathrm{r}+1}-\mathrm{u}_{\lambda}^{\mathrm{r}+1}-(\mathrm{r}+1) \mathrm{u}_{\lambda}^{\mathrm{r}} \mathrm{u}^{+}\right]
\end{gathered}
$$

(i) let $\mathfrak{u}^{+} \in W_{0}^{1, p}\left(\Omega_{a}^{+}\right)$, and for $\left\|u^{+}\right\|$sufficiently small, we have

$$
\mathrm{I}_{\lambda}(\mathrm{u}) \geq \frac{1}{\mathrm{p}} \int_{\Omega}\left|\nabla\left(\mathrm{u}_{\lambda}+\mathfrak{u}\right)\right|^{\mathrm{p}}-\left.\left.\frac{1}{\mathrm{p}} \int_{\Omega}\right|_{\Omega} \nabla\left(\mathrm{u}_{\lambda}+\mathfrak{u}^{+}\right)\right|^{p}+\frac{1}{\mathrm{p}} \int_{\Omega}\left|\nabla u_{\lambda}\right|^{p}+\int_{\Omega} \mathrm{g}_{\lambda}\left(x, u_{\lambda}\right) \mathfrak{u}^{+}
$$


then,

$$
\mathrm{I}_{\lambda}(\mathrm{u}) \geq \frac{1}{\mathrm{p}} \int_{\Omega}\left|\nabla \mathrm{u}_{\lambda}\right|^{p}+\int_{\Omega} \mathrm{g}_{\lambda}\left(\mathrm{x}, \mathrm{u}_{\lambda}\right) \mathrm{u}^{+} \geq \frac{1}{\mathrm{p}} \int_{\Omega}\left|\nabla \mathrm{u}_{\lambda}\right|^{\mathrm{p}}=\mathrm{I}_{\lambda}(0)
$$

(ii) let $v_{1} \in \mathrm{W}_{0}^{1, \mathrm{p}}\left(\Omega_{\mathrm{b}}^{+}\right), v_{1} \geq 0, v_{1} \neq \equiv 0$, such that $\int_{\Omega} \mathrm{b}(\mathrm{x}) v_{1}^{\mathrm{r}+1}>0$. We have, for large $\mathrm{s}$

$$
\begin{gathered}
\mathrm{I}_{\lambda}\left(s v_{1}\right)=\frac{1}{p} \int_{\Omega}\left|\nabla\left(u_{\lambda}+s v_{1}\right)\right|^{p}-\frac{1}{q+1} \int_{\Omega} a(x)\left[\left(u_{\lambda}+s v_{1}\right)^{q+1}-u_{\lambda}^{q+1}-(q+1) u_{\lambda}^{q} s v_{1}\right] \\
-\frac{\lambda}{r+1} \int_{\Omega} b(x)\left[\left(u_{\lambda}+s v_{1}\right)^{r+1}-u_{\lambda}^{r+1}-(r+1) u_{\lambda}^{r} s v_{1}\right] \\
=\frac{s^{p}}{p} \int_{\Omega}\left|\nabla\left(\frac{u_{\lambda}}{s}+v_{1}\right)\right|^{p}-\frac{s^{q+1}}{q+1} \int_{\Omega} a(x)\left[\left(\frac{u_{\lambda}}{s}+v_{1}\right)^{q+1}-\left(\frac{u_{\lambda}}{s}\right)^{q+1}-\frac{(q+1) u_{\lambda}^{q} v_{1}}{s^{q}}\right] \\
-\frac{\lambda s^{r+1}}{r+1} \int_{\Omega} b(x)\left[\left(\frac{u_{\lambda}}{s}+v_{1}\right)^{r+1}-\left(\frac{u_{\lambda}}{s}\right)^{r+1}-\frac{(r+1) u_{\lambda}^{r} v_{1}}{s^{r}}\right] \\
=O\left(s^{p}\right)-\frac{\lambda s^{r+1}}{r+1} \int_{\Omega} b(x) v_{1}^{r+1} \longrightarrow-\infty
\end{gathered}
$$

as $s \longrightarrow \infty$.

(iii) We now prove $I_{\lambda}(u)$ satisfies the (PS) condition in $W_{0}^{1, p}(\Omega)$. Indeed, if $\mathfrak{u}_{k}$ is a (PS) sequence, i.e. $\mathrm{I}_{\lambda}\left(u_{k}\right) \longrightarrow c, I_{\lambda}^{\prime}\left(u_{k}\right) \longrightarrow 0$. Then, for $p<\theta<r+1, \varepsilon_{k} \longrightarrow 0$, and some constant $c$, we have,

$$
\theta \mathrm{I}_{\lambda}\left(\mathrm{u}_{\mathrm{k}}\right)-\mathrm{I}_{\lambda}^{\prime}\left(\mathrm{u}_{\mathrm{k}}\right) \cdot \mathrm{u}_{\mathrm{k}} \leq \mathrm{c}+\varepsilon_{\mathrm{k}}\left\|\mathrm{u}_{\mathrm{k}}\right\|
$$

where $\left\|\mathfrak{u}_{k}\right\|$ denotes the $W_{0}^{1, p}(\Omega)$ norm $\left(\int_{\Omega}|\nabla \mathfrak{u}|^{p}\right)^{\frac{1}{p}}$.

$$
\begin{aligned}
& \left(\frac{\theta}{p}-1\right)\left\|u_{k}\right\|^{p} \leq\left(\frac{\theta}{q+1}-1\right) \int_{\Omega} a(x) u_{k}^{q+1}+\lambda\left(\frac{\theta}{r+1}-1\right) \int_{\Omega} b(x) u_{k}^{r+1}+c+\varepsilon_{k}\left\|u_{k}\right\| \\
& \left(\frac{\theta}{p}-1\right)\left\|u_{k}\right\|^{p}+\lambda\left(1-\frac{\theta}{r+1}\right) \int_{\Omega} b(x) u_{k}^{r+1} \leq\left(\frac{\theta}{q+1}-1\right) \int_{\Omega} a(x) u_{k}^{q+1}+c+\varepsilon_{k}\left\|u_{k}\right\|
\end{aligned}
$$

By $a(x), b(x)$ is bounded in $\Omega$, we obtain,

$$
\left(\frac{\theta}{p}-1\right)\left\|\mathfrak{u}_{k}\right\|^{p}+c_{2} \lambda\left(1-\frac{\theta}{r+1}\right)\left\|\mathfrak{u}_{k}\right\|^{r+1} \leq c_{1}\left(\frac{\theta}{q+1}-1\right)\left\|\mathfrak{u}_{k}\right\|^{q+1}+c+\varepsilon_{k}\left\|\mathfrak{u}_{k}\right\|
$$

since $q+1<p<r+1$, this implies that the sequence $\left(u_{k}\right)$ be bounded in $W_{0}^{1, p}(\Omega)$. Thus, from (i)-(iii), $\mathrm{I}_{\lambda}$ satisfies the assumptions of the mountain pass theorem,i.e. $\mathrm{I}_{\lambda}$ has a nontrivial critical point. This concludes the proof of Theorem 2.2.

Received: February 2012. Accepted: October 2012. 


\section{References}

[1] Francisco Odair de Paiva, Nonnegative solutions of elliptic problems with sublinear indefinite nonlinearity, J.Funct.Anal. 261(2011) 2569-2586.

[2] J.Garcia, I.Peral,J.Manfredi, Sobolev versus Hölder local minimizers and global multiplicity for some quasilinear elliptic equations, Commun.Contemp. Math. 2(3)(2000) 385-404.

[3] D.G.de Figueiredo,J.-P.Gossez,P.Ubilla, Local "superlinearity" and "sublinearity" for the pLaplacian, J.Funct.Anal. 257(2009) 721-752.

[4] Z.M.Guo,Z.T.Zhang, $W^{1, p}$ versus $C^{1}$ local minimizers and multiplicity results for quasilinear elliptic equations, J.Math.Anal.Appl. 286(2003) 32-50.

[5] Z.M.Guo, J.R.L.Webb, Uniquenss of positive solutions for quasilinear equations when a parameter is large ,Proc.Roy.Soc. Edinburgh Sect.A 124(1994) 189-198.

[6] Z.M.Guo, J.R.L.Webb, Large and small solutions of a class of quasilinear elliptic eigenvalue problems, J.Differential Equations 180(2002) 1-50.

[7] M.A. Herrero and J.L.Vazquez. On the propagation properties of a nonlinear degenerate parabolic equation, Comm. Partial Diff. Equations, (7)(1982), 1381-1402.

[8] J.R. Esteban and J.L.Vazguez. On the equation of turbulent filtration in one-dimensional porous media, Nonlinear Anal., 12(10)(1984), 1303-1325.

[9] M.Struwe, Variational Methods, Applications to Nonlinear PDE and Hamiltonial Systems, Springer-Verlag, Berlin, 1996.

[10] Z.D. Yang and B.Xu., Entire bounded solutions for a class of quasilinear elliptic equations. Boundary Value Problems 2007.Art.ID 16407, 1-8.

[11] A.V.Lair. Large solutions of mixed sublinear/superlinear elliptic equations .J.Math. Anal.Appl.346(2008) 99-106.

[12] A. V. Lair, A. W. Shaker, Entire solutions of a singular elliptic problem, J. Math. Anal. Appl., 200 (1996), 498-505.

[13] A. V. Lair, A. W. Shaker, Classical and weak solutions of a singular semi- linear elliptic problem, J. Math. Anal. Appl., 211 (1997), 371-385.

[14] C.A. Santos, On Ground state solutions for singular and semi-linear problems including superlinear terms at the infinite, Nonlinear Anal., 71 (2009) 6038- 6043.

[15] Z. Zhang, A remark on the existence of entire solutions of a singular semi- linear elliptic problem, J. Math. Anal. Appl., 215 (1997), 570-582. 
[16] Zongming Guo, Some existence and multiplicity results for a class of quasilinear elliptic eigenvalue problems, Nonlinear Anal.18(1992),957-971.

[17] Zongming Guo, On the number of positive solutions for quasilinear elliptic eigenvalue problems, Nonlinear Anal. 27(2)(1996)229-247.

[18] Zongming Guo, Existence and uniqueness of positive radial solutions for a class of quasilinear elliptic equations, Applicable Anal.47(1992),173-190.

[19] Zongming Guo, Boundary value problems of a class of quasilinear ordinary differential equations, Diff. Integral Eqns. 6(3)(1993),705-719.

[20] Zuodong Yang, Existence of entire explosive positive radial solutions for a class of quasilinear elliptic systems, J. Math. Anal.Appl.288(2003),768-783.

[21] Zuodong Yang and Q.S. Lu, Blow-up estimates for a quasilinear reaction-diffusion system, Math. Methods in the Appl.Sci.26(2003),1005-1023.

[22] Zuodong Yang and Q.S. Lu, Nonexistence of positive solutions to a quasilinear elliptic system and blow-up estimates for a quasilinear reaction-diffusion system, J. Computational and Appl.Math.50(2003),37-56.

[23] Zuodong Yang, Existence of positive entire solutions for singular and non-singular quasi-linear elliptic equation, J.Comput.Appl.Math.197(2006),355-364. 